\title{
En diálogo sobre cómo actúa Dios en el mundo. Recensión a un libro de Christoph Böttigheimer
}

Speaking about how God acts in the world. Review of Christoph Böttigheimer's book

\section{RUBÉN PEREDA}

Facultad Eclesiástica de Filosofía

Universidad de Navarra

ruben.pereda@unav.es

Palabras clave: filosofía; razón; creación; causalidad.

Keywords: philosophy; reason; creation; causality.

El profesor Böttigheimer, catedrático de Teología fundamental en la Universidad católica de Eichstätt-Ingolstad (Alemania), plantea en este ensayo $^{1}$ un estudio de las relaciones entre teología y ciencias de la naturaleza. El fin que persigue es aclarar cómo es posible que en la sociedad actual se siga defendiendo un Dios personal que actúa e interviene en el mundo. Su planteamiento es teológico, es decir, parte de la verdad revelada en la

1 Böttigheimer, Christoph. 2015. ¿Cómo actúa Dios en el mundo? Reflexiones en el marco de la tensa relación entre teología y ciencias de la naturaleza. Salamanca: Ediciones Sígueme. 
Sagrada Escritura, que presenta a Dios como un ser personal con el que el hombre puede relacionarse. Dicho de otro modo: este ensayo es, ante todo, un estudio y una defensa de la providencia divina y la religiosidad humana. En este sentido, más que una discusión de temas propiamente teológicos, es una reflexión acerca del espacio de la fe en una sociedad cuyo garante de la verdad son las ciencias de la naturaleza.

A tenor de lo dicho se advierte que Böttigheimer parte de una relación tensa: el camino del diálogo entre teólogos y científicos está plagado de obstáculos e incomprensiones mutuas que afectan, principalmente, al actuar divino en el mundo. Lo más grave de esta relación es que el Dios que presenta la fe bíblica es un Dios con una acción personal en el mundo y en la historia, „de ahí que, si ese discurso sobre el libre actuar divino pierde su fuerza de convicción, la fe que se basa en la acción reveladora de Dios en la historia queda privada de su base” (p. 10). ¿Cuáles son, en concreto, estos obstáculos? Böttigheimer se centra en su contexto más inmediato: la Alemania de comienzos del s. XXI. Así, si bien la historia de la relación entre teología y ciencias de la naturaleza todavía está pendiente de alcanzar un consenso objetivo, se encuentran en ella algunos elementos inevitables. Por ejemplo, el hecho de que desciende entre los más jóvenes la creencia en un Dios personal, o la falta de conocimiento de las verdades teológicas. A lo largo de los siglos XIX y XX, observa Böttigheimer, se da un proceso de secularización de la comprensión del yo y del mundo, hasta llegar a una situación en la que "la pujanza explicativa que emanan las ciencias de la naturaleza moderna sigue influyendo hoy de forma perdurable en la imagen del mundo del hombre moderno y, con ello, en su sensibilidad y su conducta” (p. 24). El obstáculo principal, por tanto, no es una determinada teoría científica, o un hallazgo que pueda poner en un compromiso a la fe: es un modo de ver el mundo, de estar frente a él, que excluye la creencia en un Dios personal. En este contexto cabe aceptar un Dios creador, pero se tratará de un Dios con rasgos deístas: „la acción creadora de Dios se circunscribe a la creación del mundo y [...] no se cuenta con un continuo obrar creador de Dios” (p. 28). El problema no es que Dios exista, es si el hombre se puede relacionar con Él o no. 
Para resolver este problema el autor intenta, en primer lugar, ofrecer un marco adecuado para las relaciones entre ciencia y teología. Apuesta decididamente por la complementariedad, es decir „,compatibilidad de lo que solo en apariencia es contradictorio, es decir, a la unión en un todo de dos ideas de igual valor que conciernen a diferentes ámbitos de la vida, no son derivables sin más una de otra y nunca pueden ser pensadas simultáneamente" (p. 40). Esta complementariedad permite y casi exige un diálogo en el que se tengan en cuenta los límites metodológicos de cada una de las disciplinas: las ciencias de la naturaleza „se hallan referidas a las estructura de realidad cuantificable y mesurable e investigan fenómenos singulares" (p. 45), la teología, por su parte, „se vuelve hacia el todo de la realidad del mundo y plantea la pregunta por el sentido de la existencia y los valores" (p. 46). Esta diferencia de métodos tiene sus consecuencias, en primer lugar para las ciencias de la naturaleza, que, por su propio método, se ven obligadas a excluir la consideración de Dios: Böttigheimer, con expresión fuerte, dice que es un método „necesariamente ateo”. Al mismo tiempo señala que se trata de un punto de vista que „nunca puede ser del todo neutral y objetivo" (p.48). En este punto hay que advertir que se mezclan tres cuestiones: el método científico, la actitud científica de búsqueda de la verdad y el bagaje del científico que investiga. Böttigheimer tiene presentes las tres cuestiones: la relación de las ciencias de la naturaleza con la teología es diferente según se trate del método científico, la actitud de búsqueda de la verdad o el bagaje personal del investigador. En los tres ámbitos puede darse un diálogo fructífero, pero para establecer este diálogo es pertinente una adecuada delimitación de áreas y, en su caso, un estudio de las relaciones que pueden darse entre método y actitud, actitud y bagaje, o bagaje y método.

El segundo elemento del marco es el estudio de la acción creadora de Dios: Böttigheimer señala que „toda afirmación sobre Dios implica una determinada afirmación sobre el ser humano y su mundo" (p. 70); una consecuencia inmediata si la explicación de la acción creadora ofrecida no es acorde con la imagen del hombre capaz de relacionarse con Dios. La postura deísta que se ha recogido anteriormente es sólo una de las posibilidades, 
y no la más favorable a la fe. Böttigheimer se apoya en la teología bíblica para indicar que „los relatos de la creación no describen un antes y un después, ni tampoco una primera causa en la cadena de causas, sino la relación (relatio) continua y esencial de la realidad con su fundamento" (p. 72-73). Esta relación entre la realidad y su fundamento es de un orden diferente a las relaciones entre realidades: Böttigheimer establece una distinción importante al indicar que la realidad -que podría identificarse con el mundo que conocemos mediante las ciencias de la naturaleza- es diferente de su fundamento: Dios, que sería el fundamento, no es real en el mismo sentido que lo es la „realidad”. Así se mantiene una distancia entre lo creado y el creador, distancia que es insalvable: en este contexto se alude a la teología negativa como la expresión más adecuada del conocimiento humano de Dios. Sin embargo, cabe preguntarse si hay algún modo de conocer la acción creadora que no dependa de la teología bíblica; Böttigheimer no ofrece respuesta a esta pregunta que, de contestarse afirmativamente, permitiría una vía de diálogo con las ciencias de la naturaleza.

El estudio no se desentiende de las polémicas históricas entre la ciencia y la fe (y más explícitamente, entre las ciencias y la Iglesia Católica): así, aparece el problema del modelo heliocéntrico y la cosmología desarrollada a partir de Copérnico, o la irrupción de la teoría de la evolución. Dejando de lado los detalles particulares de estos problemas, resulta muy interesante cómo dan lugar a una cosmovisión en el que parece no haber sitio para Dios: „un principio físico fundamental pasó a ocupar el lugar del Creador” (p. 85). Sin embargo, señala acertadamente Böttigheimer que esta cosmovisión parte de un concepto erróneo de la relación de Dios con el tiempo y el espacio. Para los partidarios de la cosmovisión que critica Böttigheimer, Dios sería un elemento más en el tiempo y el espacio; pero, en realidad, Dios se sitúa fuera de ambos, en un plano que no se puede captar con los métodos científico-naturales. Otra cuestión es si, aparte del método teológico, que se mueve en el ámbito de lo atemporal, hay otros modos de conocer que permitan un puente entre ambos métodos. La metafísica y la filosofía de la naturaleza, que serían dos candidatos a ocupar este puesto intermedio, no parece que tengan un lugar en el diálogo entre teología 
y ciencias de la naturaleza. Así, además del rechazo de Dios como „una causa más junto a otras causas intramundanas” (p. 118), podría ofrecerse un desarrollo de un Dios que actúa como „otra causa antes de las causas intramundanas" que completase la crítica tanto a la intrusión de las ciencias de la naturaleza en el ámbito de la teología como el intento de resolver problemas de la naturaleza mediante el conocimiento de la fe. La utilidad -e incluso necesidad- de una sólida doctrina metafísica también se hace presente al tratar de la acción de Dios en la historia: Böttigheimer acude a la noción de participación para señalar que „puesto que el esse commune, el ser común, participa en el ser verdadero, Dios está presente por doquier y es al mismo tiempo superior a todo"; sin embargo, la participación es una noción discutida, y según cómo se entienda el sentido de la participación en el ser verdadero -y de la relación con Dios, por tanto- puede variar enormemente. El autor señala que hay una desproporción infinita entre Dios y la criatura, desproporción que hay que explicar adecuadamente a la hora de introducir la participación y, como hace en otros puntos, la analogía: el peligro es que lo que se afirma por un lado -la infinita distancia-se niegue por otro. Resulta ilustrativo, en este sentido, que la explicación mediante la distinción entre causas primeras y segundas se incluya dentro del apartado dedicado a la acción mediada de Dios y su carácter personal. Así, si bien no se dice explícitamente, queda en entredicho la posibilidad de explicar la acción creadora de Dios como una relación entre diferentes sentidos de causa.

La explicación del actuar de Dios en el mundo sigue posteriormente otros derroteros, ya que se centra en la libertad humana y su relación con la libertad divina, para dirigirse paulatinamente a lo que en otros contextos se llama acción divina especial: los milagros -sobre todo los de la vida de Jesús de Nazaret y su resurrección-, la elevación sobrenatural, o la oración de petición. Se trata, efectivamente, de temas puramente teológicos, cuya aceptación en el mundo contemporáneo parece en duda. La exposición y defensa que hace Böttigheimer continúa el hilo del estudio anterior: parte de un Dios personal y libre que está fuera del tiempo. Muestra un profundo conocimiento de la Escritura y de la psicología humana, de tal forma que ofrece respuestas sólidas y convincentes. 
En resumen, la obra ¿Cómo actúa Dios en el mundo? presenta el intento de un teólogo para explicar la compatibilidad y posibilidad de diálogo entre la teología y las ciencias de la naturaleza. Trata de comprender y explicar la actitud científica que, en numerosos casos, ha llevado al rechazo de cualquier forma de teísmo. Como una muestra del carácter dialógico de este libro, cada capítulo presenta una interesante „Perspectiva”, que recoge la discusión anterior y pone las bases para un diálogo fructífero. En este sentido es un libro prometedor, que puede permitir un acercamiento sereno a las „tensa relación entre teología y ciencias de la naturaleza”. Al mismo tiempo, deja abiertas líneas de investigación que pueden ser muy fecundas: posibilidades como el desarrollo de una adecuada filosofía de la naturaleza que no sólo dialogue con las ciencias, sino que aproveche los descubrimientos de estas para un conocimiento más profundo de la realidad y más cercano a Dios, o el estudio de una metafísica de la causalidad que establezca cuáles son las relaciones entre la causa primera y las segundas no se abordan en esta obra, y son cuestiones cuya resolución es decisiva para el correcto entendimiento entre teología y ciencias de la naturaleza. 